\title{
El mercado español del bacalao en salazón: una aproximación regional desde la geografía de las convenciones.
}

\section{The salted cod market in Spain: a regional approach using the theory of conventions}

\author{
Ana Espinosa Seguî* y José Luis Sánchez Hernández**
}

\section{LA TEORÍA DE LAS CONVENCIONES COMO MARCO DE REFERENCIA PARA EL ESTUDIO DE LA GEOGRAFÍA DE LOS ALIMENTOS}

El mercado alimentario de los países europeos viene experimentando notables transformaciones durante las dos últimas décadas debido al estancamiento cuantitativo de la demanda que se deriva del lento crecimiento demográfico, a la creciente competencia entre productores de muy distintos tamaños empresariales y procedencias geográficas, y a las nuevas inquietudes y criterios que guían las decisiones de compra de los hogares y también del sector de la hostelería y la restauración, donde se efectúa ya el 29\% del gasto alimentario total en España (MARM, en línea).

En efecto, los criterios convencionales de compra, como el precio y su relación con la cantidad adquirida, no sirven ya como únicas guías para comprender la situación actual de la producción, la distribución y el consumo de alimentos. Nuevos criterios como la salubridad, la procedencia geográfica, la huella ecológica, la responsabilidad social o la imagen de marca juegan papeles diferentes en cada caso, pero relevantes en su conjunto, en la toma de deci-

\footnotetext{
* Departamento de Geografía Humana, Universidad de Alicante (Ana.Espinosa@ua.es).

** Departamento de Geografía, Universidad de Salamanca (jlsh@usal.es).
} 
siones de compra y venta de alimentos y bebidas por parte de la industria, los minoristas y el gran público (Sánchez, 2009).

Desde una perspectiva geográfica, además, no debe olvidarse que existe toda una «geografía del gusto» (Pitte, 2001) en virtud de la cual los consumidores de distintas regiones y países europeos manifiestan preferencias (y rechazos) bien definidas por determinados tipos de alimentos o de preparaciones de los mismos, lo que no hace sino añadir complejidad a las estrategias de los grandes productores, a la vez que habilita nichos de mercado, en ocasiones muy rentables, para los pequeños elaboradores locales más especializados.

Para interpretar de forma integrada la relación entre estos procesos y sus implicaciones para el crecimiento, la reestructuración o la desarticulación de las cadenas de suministro de alimentos, la Geografía Económica ha recurrido a la llamada «teoría de las convenciones», originaria de Francia (Boltanski y Thévenot, 1991) y aplicada al estudio del mercado alimentario en el Reino Unido desde finales de la década de 1990 (Murdoch y Miele, 1999; Murdoch, Marsden y Banks, 2000). Desde entonces, este marco conceptual ha sido utilizado para dar un sentido de coherencia e interrelación a fenómenos tan pujantes y tan diversos como la agricultura orgánica (Rosin y Campbell, 2009), la recualificación del sector vitivinícola (Guthey, 2008; Ponte, 2009; Sánchez, Alonso y Aparicio, 2010), los mercados callejeros de productos frescos (Kirwan, 2006), los huertos urbanos (Pudup, 2008), el comercio justo (Renard, 2003), las agro-cadenas globales de valor (Ponte y Gibbon, 2005) o las dietas relacionadas con el estilo de vida (Morris y Kirwan, 2007), por citar algunas contribuciones relevantes.

La aplicación a la Geografía Económica, y en particular al estudio del mercado alimentario, de la teoría francesa de las convenciones se explica por el carácter comprensivo de esta construcción conceptual. Boltanski y Thévenot (1991) aúnan la Sociología y la Economía en su estudio de la empresa industrial capitalista como organización que debe combinar continuamente intereses distintos e incluso contrapuestos: fabricar productos fiables, contener los costes de producción, ampliar su cuota de mercado, desarrollar nuevas líneas de negocio, preservar su reputación, retener el talento de sus empleados, contribuir al bienestar de su comunidad... Según estos autores, incluso en una sociedad organizada conforme a los principios de la propiedad privada y la libertad de mercado, la gestión empresarial no puede perseguir únicamente la maximización del beneficio, sino que su pertenencia a un contexto territorial complejo de vínculos y relaciones sociales, políticas, culturales y económicas le obliga a construir un compromiso permanente e inestable entre diversas lógicas que califican como «industriales», «comerciales», «domésticas», «públicas», «cívicas»y «ecológicas», incorporadas estas últimas en textos posteriores. 
La transposición de este sugerente marco teórico a los estudios geográficos sobre la producción, distribución y consumo de alimentos se apoya en la ya citada evidencia de que la evaluación o valoración de los alimentos en los países avanzados se ha vuelto más compleja durante los últimos años. La noción de calidad ya no está ligada en exclusiva a la relación entre el precio y las propiedades organolépticas de los alimentos (sabor, color, aroma, textura), sino que en su definición intervienen nuevos criterios o «convenciones» que condicionan las elecciones de los consumidores y, por tanto, orientan las decisiones de los productores.

Las convenciones industriales evalúan los alimentos según los estándares técnicos de procesado, higiene y conservación que se les han aplicado durante su manipulación y distribución. Las convenciones comerciales se refieren a la utilidad y el precio de los alimentos. Las convenciones domésticas confieren importancia a lo local, ensalzando las formas tradicionales de elaboración y atribuyendo a los productos locales valores de calidad y de arraigo muy apreciados por los consumidores más exigentes. Las convenciones públicas derivan de la reputación o reconocimiento de las marcas, distintivos y envases entre los consumidores. Las convenciones cívicas valoran ante todo la contribución de los procesos y productos alimenticios al bienestar individual y colectivo de todos los ciudadanos. Las convenciones ecológicas, por último, se interesan por el impacto ambiental del proceso de elaboración de cada alimento. En definitiva, las convenciones constituyen «...acuerdos que guían la acción (económica), que es el resultado de una percepción compartida del entorno (económico)» (Parrott, Wilson y Murdoch, 2002, p. 244) y la teoría de las convenciones, en su conjunto, «...se interesa por las características de las personas y de los bienes que concurren en el mercado y por la forma en que interaccionan para coordinar los intercambios» (Kirwan, 2006, p. 303), que comprende el mecanismo de los precios, pero también otros elementos de juicio y evaluación, hasta el punto de que Wilkinson (2002, p. 344) la considera como «un marco analítico muy útil para explorar la negociación de intereses y valores diferenciados».

Así pues, para cada producto (alimento, en este caso), en un momento y lugar dados, prevalece un conjunto o «coalescencia» (Rosin, 2008) específica de convenciones donde los distintos tipos citados ocupan posiciones desiguales o pueden incluso quedar ausentes en algunos casos. Estas coalescencias de convenciones expresan consensos forjados en el seno de una red de productores y consumidores sobre las propiedades o cualidades que debe reunir un determinado producto. Tanto o más que las restantes actividades económicas, la agroindustria muestra una marcada tendencia a la concentración local y la es- 
pecialización regional por motivos naturales e históricos. De ahí que pueda formularse la hipótesis de la existencia de una geografía regional de las convenciones que definen la calidad de los alimentos.

En efecto, los espacios puramente locales carecen del tamaño y el poder suficientes para influir en la re-definición de las convenciones a lo largo de la red de producción-distribución-consumo, donde intervienen numerosos agentes externos, tanto económicos como políticos. En el otro extremo, los grandes conjuntos subcontinentales (Parrott, Wilson y Murdoch, 2002; Galdós, 2004) son demasiado extensos y heterogéneos para efectuar estudios detallados. Incluso la escala nacional parece imprecisa: Amilien et al. (2007), por ejemplo, comparan la valoración que los consumidores urbanos hacen de los alimentos locales protegidos en Francia y Noruega, pero sus resultados se limitan a reproducir el conocido contraste cultural entre el norte y el sur de Europa.

En cambio, la escala regional de análisis puede enriquecer notablemente la investigación geográfica sobre las convenciones alimentarias porque la región proporciona el marco de elaboración y transformación en la definición de la calidad de los alimentos. La Geografía Económica ha recuperado la región como unidad de análisis a partir de los trabajos de Storper (1995 y 1997) que la re-definen como un espacio de relaciones formales e informales entre los agentes económicos. La acción económica genera y transforma las condiciones materiales e institucionales locales y produce entornos regionales diferenciados; éstos, a su vez, actúan como contextos específicos que influyen en las decisiones y estrategias de los agentes. En esta línea, Lagendijk (2004) sostiene que la región constituye la escala adecuada para el estudio de las relaciones entre economía y cultura, donde las convenciones juegan un papel principal porque son imprescindibles para el funcionamiento de las redes sociales y económicas que articulan la región y la conectan con otros territorios.

En este contexto teórico, el presente artículo se plantea dos objetivos principales. Primero, trazar una geografía regional de las convenciones que definen la calidad del bacalao en salazón en una muestra amplia y representativa de las regiones españolas. Segundo, averiguar si en la última década se han registrado transformaciones relevantes en la coalescencia de convenciones específica de cada región. En caso afirmativo, cabe plantear tres preguntas adicionales: ¿qué agentes o factores han generado tales cambios?; ioperan los cambios a escala nacional o las regiones muestran evoluciones específicas?; ¿qué regiones conservan su definición tradicional de calidad para el bacalao en salazón y cuáles han remodelado su particular coalescencia de convenciones?

Con este propósito, el resto del artículo se estructura en cuatro apartados. La sección segunda describe el contexto institucional en que se inserta esta in- 
vestigación, expone las fuentes de información consultadas y presenta la metodología utilizada. La sección tercera presenta de forma sucinta el papel del bacalao en salazón en la cultura alimentaria española. La cuarta sección describe las convenciones imperantes en las comunidades autónomas investigadas. La quinta discute los resultados regionales para identificar los principales cambios identificados, los factores que los propician y los agentes que los impulsan, así como las modulaciones regionales de todos estos elementos. Por último, las conclusiones sintetizan los resultados principales del trabajo.

\section{Metodología y FUENTES DE INFORMACiÓN}

Esta investigación se desarrolló en el seno del proyecto "The Spanish salted fish market and the opportunities for the Norwegians" (2008-2011), financiado por la Fundación Noruega para la Ciencia (Norges forskningsråd) y coordinado por las universidades de Bergen y Tromsø, en el cual participaron las universidades españolas de Alicante, Salamanca y Sevilla. Su objetivo principal era la identificación de las convenciones que definen la calidad del bacalao salado en los principales mercados regionales españoles. Se partía, por tanto, de la hipótesis de que España no puede ser considerada un territorio homogéneo en cuanto a la elaboración, comercialización y consumo de un producto tan arraigado en su cultura gastronómica como el bacalao salado, tal y como sugerían diversos trabajos anteriores que ponen de relieve la diversidad regional de la tradición bacaladera española (Lindkvist y Lois, 2008; Gallart, Escriche y Fito, 2005; Gallart, Heide y Ostli, 2005).

La identificación de las convenciones que definen un bacalao de calidad se centró en las principales regiones productoras, comercializadoras o consumidoras, que son el País Vasco, Galicia, Andalucía, Madrid, Cataluña y la Comunidad Valenciana. Para estas seis comunidades autónomas, que concentran 55 de las 67 empresas elaboradoras o importadoras registradas en España en 2009, se recopiló información estadística y también información cualitativa, mediante trabajo de campo, conforme al procedimiento que se describe a continuación.

La información cuantitativa procede de la Base de Datos de Consumo en Hogares que elabora el Ministerio de Agricultura, Alimentación y Medio Ambiente y que ofrece datos de consumo de pescado por comunidades autónomas, pero sin desagregar la categoría de pescado salado en sus distintas especies. Por ese motivo, en regiones de fuerte tradición consumidora de otros pescados en salazón como Andalucía o la Comunidad Valenciana, los resultados sobre consumo regional deben ser tratados con cierta cautela. 
Las limitaciones de estos datos oficiales en el plano cualitativo (sabor, textura, color, preparación, presentación, origen geográfico) hicieron preciso el diseño de entrevistas semiestructuradas con los principales agentes implicados en la importación, transformación, distribución, elaboración y consumo del bacalao en salazón en cada comunidad autónoma. Se efectuaron 187 entrevistas conforme a la distribución que figura en el cuadro 1.

En primer lugar, los elaboradores e importadores de bacalao salado son claves en la conformación de las convenciones que modulan la construcción social de la calidad. Por ese motivo, se centró gran parte del estudio cualitativo en la visión que estos agentes tienen sobre la evolución del papel que corresponde al bacalao salado en la gastronomía y consumo de su área de mercado. De un total de 55 empresas en activo contabilizadas en el año 2009 en estas seis regiones, se entrevistó a 34 compañías, lo que supone dos tercios del universo considerado para el estudio. Como la mayor parte de la producción y el consumo de bacalao en España se concentran en estas regiones, los resultados obtenidos se consideran lo suficientemente fiables para representar y analizar el mercado nacional del bacalao salado. Las entrevistas se centraron en conocer la procedencia geográfica del bacalao importado, el área de distribución

\section{Cuadro 1}

DISTRIBUCIÓN DE LAS ENTREVISTAS EN PROFUNDIDAD

A LO LARGO DE LA CADENA DE VALOR

\begin{tabular}{lcccccc}
\hline Comunidad & $\begin{array}{c}\text { Empresas } \\
\text { en activo } \\
\text { Autónoma }\end{array}$ & \multicolumn{5}{c}{ Entrevistas a: } \\
\cline { 3 - 7 } & $22009)$ & Empresas & $\begin{array}{c}\text { Comercios } \\
\text { Minoristas }\end{array}$ & Restaurantes & Consumidores & Total \\
\hline País Vasco & 22 & 9 & 7 & 7 & 7 & 30 \\
Galicia & 9 & 6 & 10 & 10 & 10 & 36 \\
Andalucía & 5 & 5 & 10 & 10 & 10 & 35 \\
Madrid & 3 & 3 & 10 & 10 & 10 & 33 \\
Cataluña & 7 & 5 & 7 & 7 & 7 & 26 \\
C. Valenciana & 9 & 6 & 7 & 7 & 7 & 27 \\
Total & 55 & 34 & 51 & 51 & 51 & 187 \\
\hline
\end{tabular}

Fuente: Memoria de investigación del Proyecto "The Spanish salted fish market". 
comercial de las empresas y el tipo de productos comercializados. En su calidad de conocedores del mercado español, y desde su posición estratégica entre la oferta y la demanda, se les preguntó también por las posibles diferencias entre las convenciones imperantes en las regiones españolas, así como por su propia experiencia en la introducción de innovaciones de producto o proceso que afectan a las convenciones imperantes en su mercado habitual.

Además, se aplicaron guiones específicos de entrevistas para los comerciantes minoristas, los restaurantes y los consumidores. El comercio minorista pone en contacto a los productores nacionales con los consumidores finales y las entrevistas revelan su función de correas de transmisión de la información y de las convenciones que consumidores y productores elaboran sobre el bacalao en salazón. Los restaurantes son lugares de elaboración y consumo que resultan fundamentales para el mantenimiento de las recetas y para la introducción de innovaciones gastronómicas; constituyen, además, el único canal donde los consumidores menos experimentados en las técnicas culinarias tradicionales pueden consumir el bacalao salado. Las respuestas de los consumidores están condicionadas por su edad y sexo tanto como por su lugar de residencia, ya que los informantes se seleccionaron en los puntos de venta, frecuentados sobre todo por mujeres mayores de cincuenta años: se les preguntó acerca de la relevancia del bacalao en la cocina local y regional, de las convenciones del mercado local y de la influencia de los nuevas presentaciones de bacalao en el mantenimiento de las recetas tradicionales y en la posible difusión de nuevos modos de preparación.

Respecto al número de entrevistas, el diseño original de la investigación incluyó algunas entrevistas adicionales en Navarra y La Rioja (para complementar el caso de estudio del País Vasco), en Aragón (como extensión del mercado catalán) y en Murcia (para ampliar el caso valenciano), con la finalidad de ofrecer una visión más amplia de España a los productores noruegos involucrados en el estudio de mercado. Sin embargo, en este artículo se ha prescindido de estas regiones «complementarias» porque sus diferencias geográficas con las respectivas regiones «centrales» son lo suficientemente relevantes como para desaconsejar un análisis conjunto de sus convenciones, toda vez que los gustos en alimentación están muy condicionados por los factores culturales (transmitidos por la lengua, entre otros vehículos) y por la influencia del medio físico (clima, relieve, acceso al mar) en las disponibilidades alimentarias ${ }^{1}$.

\footnotetext{
${ }^{1}$ Agradecemos esta importante precisión a un evaluador anónimo del artículo.
} 


\section{El bacalao en la CUltura Alimentaria española}

Con 42,9 kilos por persona en el año 2009, último disponible en FAOSTAT (FAO) [en línea], España era el país de la Unión Europea con mayor consumo relativo de pescado, seguido de lejos por Francia $(33,7 \mathrm{~kg}$ ), y el sexto del mundo, tras Islandia $(88,3 \mathrm{~kg})$, Japón $(56,6 \mathrm{~kg})$, Corea $(56,1 \mathrm{~kg})$, Malasia $(53,2 \mathrm{~kg})$ y Noruega $(50,6 \mathrm{~kg})$. Aunque estas cifras parecen apuntar, en la escala macro, hacia una estrecha relación entre acceso al mar y consumo de pescado, el desglose regional para el caso español desmiente parcialmente esta apreciación. Las comunidades cantábricas se sitúan en cabeza (Asturias, Cantabria y el País Vasco), pero las regiones interiores como Castilla y León (de forma muy destacada), Madrid, Aragón o Castilla-La Mancha superan los datos de todas las comunidades mediterráneas mientras, paradójicamente, ambos archipiélagos presentan las cifras más bajas (figura 1).

FIGURA 1

CONSUMO DE PESCADO POR HABITANTE EN HOGARES, 2011 (KILOS)

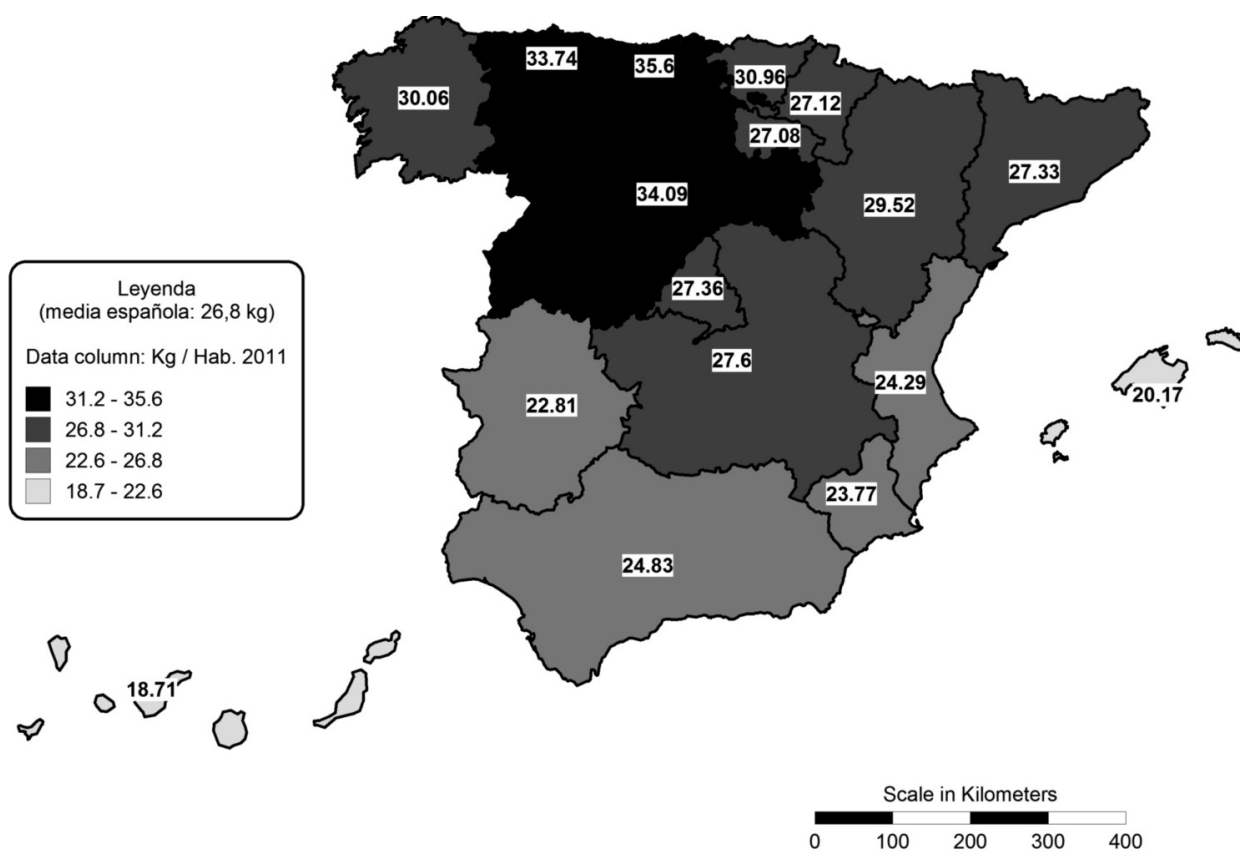

Fuente: elaboración propia a partir de Base de Datos de Consumo en Hogares.

Estudios Geográficos, Vol. LXXIV, 275, pp. 439-467, julio-diciembre 2013

ISSN: 0014-1496, eISSN: 1988-8546, doi: 10.3989/estgeogr.201316 
El bacalao representa el 5,9\% del volumen y el 6,6\% en valor del pescado consumido en España durante 2012 (cuadro 2). Esas cifras se dividen entre las presentaciones frescas y congeladas en una relación 55\%-45\%, muy lejos de la supremacía indiscutible del pescado fresco ( $80 \%$ de volumen y gasto) en su conjunto, y ello pese a que el precio medio del bacalao congelado supera levemente al del producto fresco.

CuAdro 2

CONSUMO DOMÉSTICO DE PESCADO EN ESPAÑA (2012)

\begin{tabular}{lccccc}
\hline & $\begin{array}{c}\text { Volumen } \\
\text { (miles de kg.) }\end{array}$ & $\begin{array}{c}\text { Valor } \\
(\text { miles de } €)\end{array}$ & $\begin{array}{c}\text { Precio medio } \\
\text { por Kg. (en } €)\end{array}$ & $\begin{array}{c}\text { Consumo per } \\
\text { cápita (en kg) }\end{array}$ & $\begin{array}{c}\text { Gasto per } \\
\text { cápita (en } €)\end{array}$ \\
\hline $\begin{array}{l}\text { Pescado y } \\
\text { marisco }\end{array}$ & $1.215 .005,13$ & $8.856 .755,59$ & 7,29 & 26,37 & 192,25 \\
\hline $\begin{array}{l}\text { Pescado } \\
\text { Pescado } \\
\text { fresco }\end{array}$ & $685.632,80$ & $4.609 .355,05$ & 6,72 & 14,88 & 100,04 \\
\hline $\begin{array}{l}\text { Pescado } \\
\text { congelado }\end{array}$ & $144.745,69$ & $917.820,76$ & 6,34 & 3,13 & 19,92 \\
\hline $\begin{array}{l}\text { Bacalao } \\
\text { total) }\end{array}$ & $40.485,91$ & $303.098,24$ & 7,49 & 0,88 & 6,57 \\
\hline $\begin{array}{l}\text { Bacalao } \\
\text { fresco }\end{array}$ & $23.284,30$ & $165.401,02$ & 7,10 & 0,51 & 3,62 \\
\hline $\begin{array}{l}\text { Bacalao } \\
\text { congelado }\end{array}$ & $17.201,61$ & $137.697,22$ & 8,00 & 0,37 & 3,01 \\
\hline
\end{tabular}

Fuente: elaboración propia a partir de Base de Datos de Consumo en Hogares.

El pescado en salazón (cuadro 3), por su parte, experimenta una notable pérdida de posiciones en el mercado español hasta su actual aportación del $2,2 \%$ en volumen y el 3,7\% en valor de las ventas. Este retroceso se ha acelerado durante la crisis económica como consecuencia de su elevado precio medio. Aunque no se dispone de datos desagregados por especies para este tipo de elaboración, que incluye también el atún o la anchoa, cabe suponer que el bacalao salado sigue la misma tendencia regresiva. 
Cuadro 3

CONSUMO DOMÉSTICO DE PESCADO EN SALAZÓN EN ESPAÑA

\begin{tabular}{|c|c|c|c|c|c|}
\hline Año & $\begin{array}{c}\text { Volumen } \\
\text { (Miles de kg) }\end{array}$ & (Miles de $€$ ) & $\begin{array}{l}\text { Valor Precio } \\
\text { medio por Kg. } \\
\quad(\text { en } €)\end{array}$ & $\begin{array}{c}\text { Consumo } \\
\text { per capital } \\
\text { (en kg) }\end{array}$ & $\begin{array}{c}\text { Gasto } \\
\text { per capital } \\
(\text { en } €)\end{array}$ \\
\hline 2005 & $22,898.79$ & $195,933.59$ & 8,56 & 0,54 & - \\
\hline 2006 & $21,791.65$ & $209,265.51$ & 9,60 & 0,51 & - \\
\hline 2007 & $19,414.30$ & $198,058.13$ & 10,20 & 0,44 & - \\
\hline 2008 & $19.099,68$ & $214.749,01$ & 11,24 & 0,44 & 4,92 \\
\hline 2009 & $18.344,01$ & $210.496,37$ & 11,47 & 0,41 & 4,68 \\
\hline 2010 & $16.943,34$ & $186.733,63$ & 11,02 & 0,37 & 4,06 \\
\hline 2011 & $15.432,48$ & $174.595,40$ & 11,31 & 0,35 & 3,60 \\
\hline 2012 & $13.881,75$ & $160.693,04$ & 11,58 & 0,32 & 3,49 \\
\hline
\end{tabular}

Fuente: elaboración propia a partir de Base de Datos de Consumo en Hogares.

No obstante, la paulatina sustitución del pescado en salazón por las presentaciones fileteadas, bien frescas, refrigeradas o congeladas, se viene observando desde mediados de la década de 1980, según distintos estudios (Gallart et al. 2003; Gallart, Heide y Ostli, 2005; Grafe, 2004; García Santos, 2007; Lindkvist, Gallart y Stabell, 2008; Lindkvist y Sánchez, 2008; Xie y Myrland, 2010; Díaz y Gómez, 2010; Sánchez, 2011a) que citan diversos factores explicativos que se complementan entre sí. Primero, la reducción de precios del pescado fresco derivada de la creciente capacidad de captura y conservación en frío de la flota de altura, junto con el aumento del poder adquisitivo y del equipamiento técnico de los hogares españoles (frigoríficos, congeladores). Segundo, la complejidad de la elaboración de recetas con pescado en salazón, que requiere un lento proceso de desalado que los consumidores urbanos rechazan por falta de tiempo y por la aparición de alternativas más sencillas y rápidas como los platos precocinados. Tercero, la difusión de las ideas que vinculan salud y dieta y que recomiendan limitar el consumo de sal por su relación con las dolencias coronarias, en particular la hipertensión. Cuarto, la sobreexplotación de los caladeros de bacalao en el Mar del Norte y la imposi- 
ción de cuotas de capturas cada vez más reducidas que limitan la oferta, elevan los precios y dificultan la competencia con otros productos más asequibles. Quinto, la reticencia de los proveedores noruegos tradicionales a modernizar sus estructuras productivas e introducir innovaciones (empaquetado, fileteado) que habrían facilitado la introducción del pescado en salazón en los nuevos canales comerciales minoristas de masas (supermercados e hipermercados).

Como consecuencia de la acción combinada de estos factores, el bacalao en salazón ha pasado de ser un alimento popular muy difundido entre consumidores de poder adquisitivo medio y bajo y también en el medio rural, a convertirse en una materia prima de precio bastante superior al promedio del resto de pescados (comparar, a este respecto, la cuarta columna de los cuadros 2 y 3), demandada por los hogares urbanos cuya cultura gastronómica y nivel de renta permiten mantener un estilo de consumo más cuidado, con mayor presencia de las recetas tradicionales muy del gusto de la población que, por su edad, conserva el recuerdo de los platos de bacalao en salazón.

Un segundo reducto de pervivencia del bacalao en salazón es la restauración de cierta calidad, donde se conservan las tradiciones culinarias en las regiones más apegadas a este producto, caso del País Vasco o Galicia (García Santos, 2007). Esta identificación del bacalao en salazón con la cocina tradicional le confiere una imagen pública positiva, como elemento integrante de la identidad local, pero también limita su consumo, que termina por ser asociado a las ocasiones especiales y a procedimientos de elaboración de cierta complejidad.

Por último, hay que mencionar la incidencia de la tradición religiosa de la Cuaresma en el consumo de bacalao, que sigue acusando cierta estacionalidad como consecuencia del cumplimiento, cada vez menos extendido y riguroso, del mandato católico de no comer carne durante determinados días de dicho período del año litúrgico. Este hecho es comúnmente citado como la causa última del arraigo del bacalao en la cultura gastronómica de la Península Ibérica y de su extensa difusión en las áreas rurales por su fácil conservación en salazón: «Los salazones, especialmente el bacalao, se consumían en grandes cantidades, así como los chicharros o el escabeche, conservas vegetales y frutos secos» (Gago, 2007, p. 151), hasta el punto de que las cartillas de racionamiento de posguerra incluían una ración de 75 gramos de bacalao salado.

La industria bacaladera española ha tenido que adecuarse a todos estos cambios en los patrones de consumo (cuadros 4 y 5). La presentación habitual de la bacalada o mariposa entera a granel, amarillenta, muy sazonada y con un período de curación de hasta cuatro meses, elaborada con bacalao noruego o 


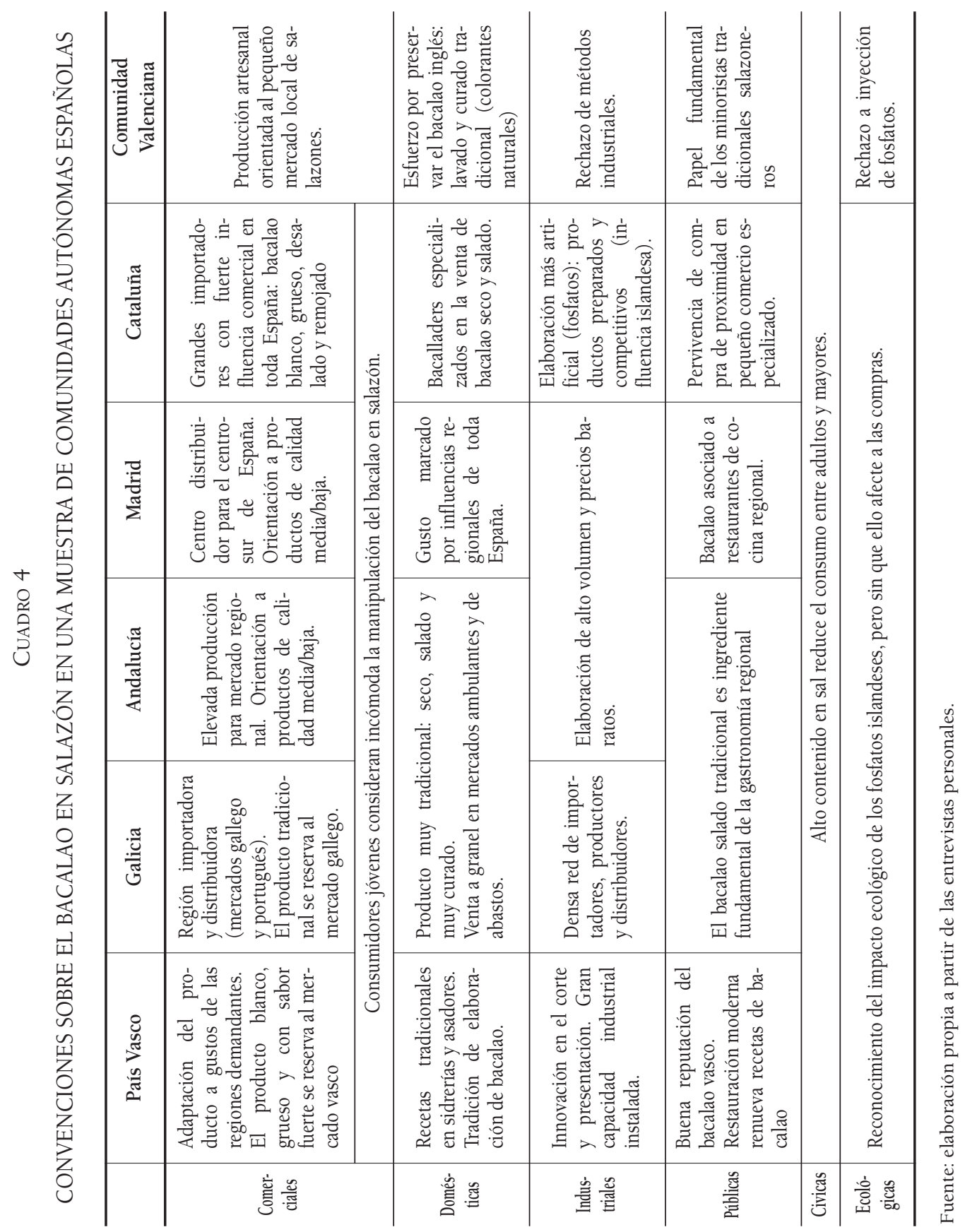

Estudios Geográficos, Vol. LXXIV, 275, pp. 439-467, julio-diciembre 2013 ISSN: 0014-1496, eISSN: 1988-8546, doi: 10.3989/estgeogr.201316 


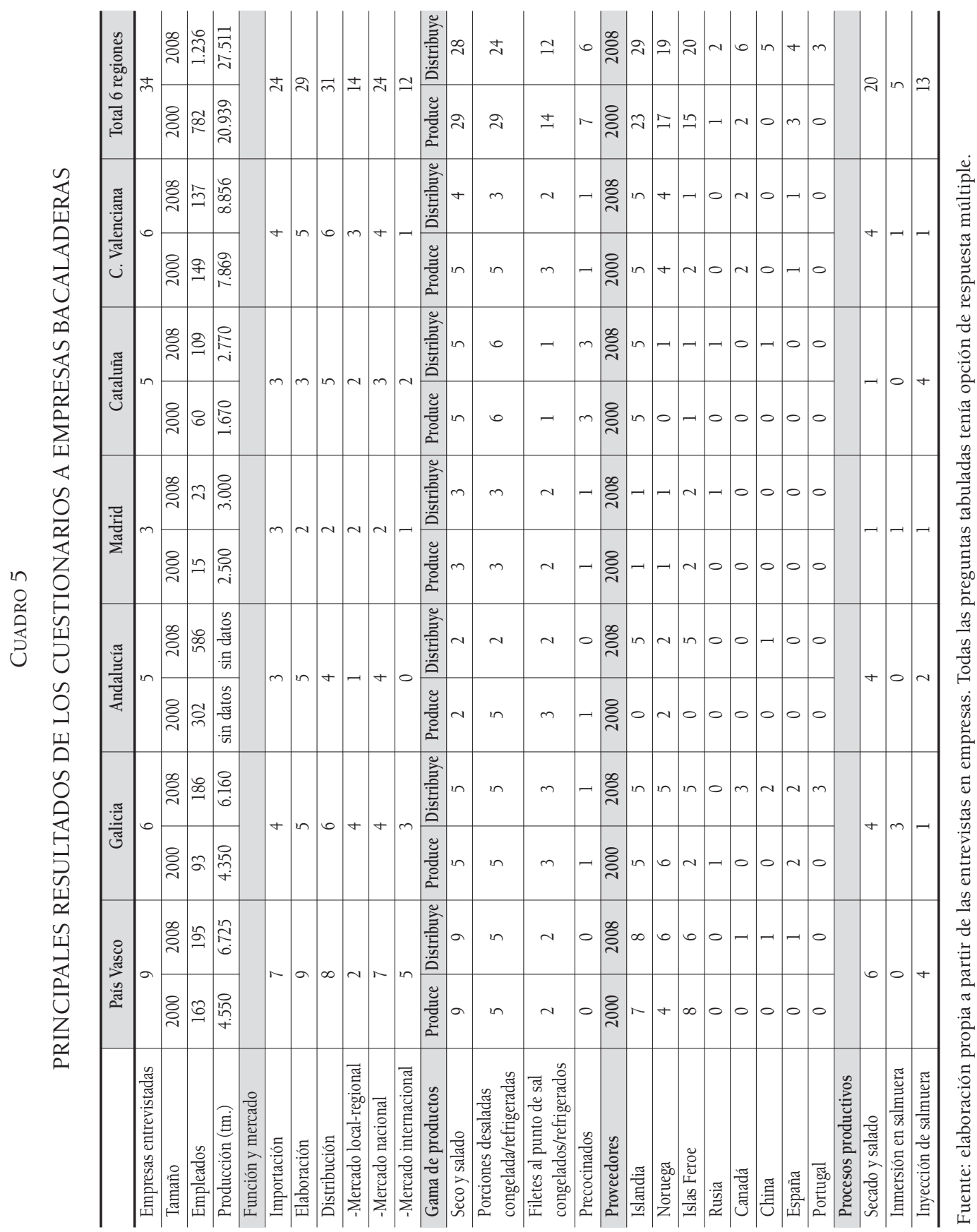

Estudios Geográficos, Vol. LXXIV, 275, pp. 439-467, julio-diciembre 2013 ISSN: 0014-1496, eISSN: 1988-8546, doi: 10.3989/estgeogr.201316 
nacional, ha dejado paso a nuevas presentaciones congeladas o refrigeradas (filetes al punto de sal, bacalao desalado, envasado y troceado), más fáciles y rápidas de cocinar, elaboradas por lo general con materia prima suministrada por nuevos proveedores con precios más bajos (China, Rusia, Canadá) o mayor orientación al mercado (Islandia, Islas Feroe). La distribución del mercado de bacalao entre el fresco y el congelado, ya mencionada por su acusada disparidad con el promedio nacional, es la consecuencia de todos estos cambios en la oferta y la demanda.

Estas tendencias generales, no obstante, adquieren rasgos específicos y distintivos en los diferentes mercados regionales españoles como consecuencia de la desigual combinación de convenciones en cada región. El siguiente apartado está dedicado precisamente a su descripción y explicación.

\section{Principales Resultados POR REGiOneS}

Las convenciones que rigen la evaluación de la calidad del bacalao salado en España muestran una notable variedad geográfica. Mientras ciertas regiones se aferran a una coalescencia muy tradicional de convenciones, otras áreas muy consumidoras han aceptado nuevas presentaciones y preparaciones más acordes con las tendencias actuales de consumo. Para evaluar esta diversidad, se presenta a continuación un análisis de los resultados de cada una de las comunidades autónomas contempladas en este trabajo, a partir del contenido del cuadro 4, que sintetiza los resultados de las entrevistas, y del cuadro 5, que tabula los datos obtenidos mediante cuestionarios a las empresas del sector.

\subsection{País Vasco}

El País Vasco posee el mayor número de empresas dedicadas a la importación, producción y comercialización de bacalao salado de todo el país (cuadro 5), cubriendo una extensa área de mercado que incluye toda España y también Francia y los países lusófonos (Portugal, Brasil y Angola), que comparten la preferencia por el bacalao más tradicional. Ello es reflejo de una herencia comercial y cultural que se remonta al siglo $\mathrm{xV}$, cuando los pescadores vascos organizaron una cadena estable de suministro de bacalao a la Península Ibérica (Kurlansky, 1999 y García Orellán, 2006) a través del puerto de Pasajes, de modo que Guipúzcoa constituye, todavía hoy, uno de los mayores centros industriales y logísticos de este producto en España. 
En esta región, el bacalao seco es muy consumido en el hogar, siguiendo con frecuencia el habitual proceso de desalado en agua, pero, sobre todo, goza de gran implantación en el ámbito de la restauración (García Santos, 2007; Espinosa y Martínez, 2011a). En su vertiente más tradicional o doméstica, los asadores y sidrerías siguen ofreciendo el bacalao como reclamo de sus menús. A su vez, la cocina vasca de autor se ha nutrido de estas recetas típicas de bacalao para rediseñarlas al gusto contemporáneo e incorporar así la reputación de este pescado al segmento de la alta gastronomía, que tanto ha contribuido a la recuperación del sector turístico vasco durante la última década.

Todas las empresas vascas elaboran y distribuyen el bacalao tradicional seco y salado, pero algunas también satisfacen la demanda de otras regiones españolas, ajustando el género a los gustos particulares de cada una (Espinosa y Martínez, 2011a y 2011b). Los productores, cocineros y consumidores del País Vasco han conformado unas convenciones domésticas basadas en las propiedades de las piezas de mayor tamaño, grosor y blancura, semejantes al aspecto de la merluza, el pescado de mejor reputación en la cocina vasca. Sin embargo, la industria guipuzcoana se dedica también a presentaciones de menor grosor y tamaño y precios más asequibles (desalados, fileteados) elaboradas con inyección de salmuera. Esa diversidad de la oferta se plasma en la ampliación del mercado de abastecimiento, que mantiene las referencias tradicionales (Noruega, Islandia, Islas Feroe), pero se ha abierto a proveedores de bajo coste como Alaska, China o Canadá.

\subsection{Galicia}

Galicia ha concentrado gran parte de la flota bacaladera española, siendo en la actualidad la región donde perviven los últimos bacaladeros en activo del país (Lindkvist y Lois, 2008; González, 2011), junto con los matriculados en el puerto guipuzcoano de Pasajes. Se explica así que en ambas regiones se emplee el bacalao español como materia prima (cuadro 5). El bacalao es un ingrediente básico de su acervo gastronómico, pero también de una cultura y una economía ligadas al mar. En ausencia del revulsivo innovador que supuso la alta cocina en el caso vasco, Galicia ha mantenido íntegras las convenciones domésticas tradicionales del bacalao en salazón, bien salado y mucho más seco que en otras regiones: cinco de las seis empresas entrevistadas declaran elaborar este producto con género portugués y escandinavo conforme al proceso tradicional.

Esta tradición se deja sentir también en las pautas de distribución y consumo. En Galicia se sigue comprando, tanto en el canal tradicional como en 
las cadenas de distribución, la bacalada entera sin ningún procesado, sin desalar, cortar o quitar las espinas. Además, la gastronomía regional se ha resistido a la sustitución de los productos típicos por otros nuevos que hubiesen cambiado no sólo las preferencias regionales de productores y consumidores, sino la propia cultura culinaria gallega. La especialización en esta gama clásica se deja sentir también en los mercados exteriores atendidos desde Galicia (Portugal y Brasil).

En este apego a las convenciones más tradicionales ha sido decisiva la localización geográfica (Espinosa y Martínez, 201la). Su cercanía con Portugal se deja sentir en la semejanza con los gustos imperantes en el país vecino y, según algunos interlocutores, esa semejanza se acentúa con la proximidad geográfica: "tenemos clientes diferentes atendiendo a su cercanía con Portugal. Mientras que la gente de Pontevedra prefiere el bacalao muy seco y salado, en La Coruña lo prefieren con tres cuartos de curación".

No obstante, las firmas gallegas, como las vascas, también aprovechan las oportunidades del resto del mercado español, incorporando elaboraciones más sencillas (desaladas, fileteadas, precocinadas), procesos más rápidos y baratos (inmersión en salmuera) y materias primas de proveedores alternativos como China o Canadá.

\subsection{Andalucía}

El mayor mercado regional de España en términos demográficos conserva las elaboraciones tradicionales del bacalao salado, cuyas variantes más curadas y sazonadas han sido durante mucho tiempo la única especie de pescado consumida en sus comarcas interiores y una fuente importante de proteínas en su dieta. Se explica así la persistencia de una estructura empresarial compuesta por cuatro pequeñas compañías que usan, sobre todo, procesos tradicionales y abastecen sobre todo al mercado andaluz, más una quinta firma de nueva instalación y grandes dimensiones que emplea técnicas más industrializadas y distribuye sus productos en el resto de España (Martínez y González, 2012).

El producto tradicional, amarillento y muy salado, correspondiente a estas convenciones domésticas, se elaboraba con materia prima noruega, también reconocida entre los interlocutores entrevistados por su superior calidad. Pero desde 2000 han ganado presencia los proveedores de Islandia, Islas Feroe y, sobre todo, China (cuadro 5), con género más barato, más blanco, menos curado y más homogéneo en el tiempo, lo que facilita su posterior adaptación a diferentes usos y presentaciones por parte de las compañías procesadoras lo- 
cales. Esta combinación de convenciones industriales y comerciales se impone poco a poco frente al género noruego, menos versátil en cuanto a sus posibilidades de manipulación y transformación.

Como se ha dicho, predomina en Andalucía el consumo del bacalao seco salado tradicional que se desala en el hogar antes de ser cocinado. Además, algunas compañías ofrecen productos de mayor precio unitario y con marca propia (desalados, filetes) orientados al mercado de la restauración y a los nuevos hábitos de consumo marcados por la rapidez y la comodidad (Consejería de Agricultura y Pesca, 2009). No obstante, su número se ha reducido desde 2000 porque las pequeñas firmas innovadoras que comenzaron a trabajar en esta dirección la han abandonado ante la incapacidad para competir con los precios de la nueva empresa, muy orientada a la gran distribución.

\subsection{Madrid}

La única región completamente interior analizada en este artículo es, a la vez, el mayor mercado pesquero de España en valor de las ventas (Espinosa y Martínez, 2011a). De cara a los objetivos de este trabajo, la característica más definitoria de Madrid es la convivencia de convenciones e influencias gastronómicas procedentes de toda España que se genera a partir de los numerosos restaurantes típicos especializados en las distintas cocinas regionales.

El número de productores de bacalao salado en Madrid es reducido y su producción se enfoca al abastecimiento de todo el centro y sur de España, con procesos de elaboración y presentaciones comerciales de todos los tipos que distingue el cuadro 5 . El bacalao de origen escandinavo (convenciones domésticas relativas al origen de los alimentos) se destina a la hostelería de cocina regional, que demanda una materia prima bien adaptada a la imagen de la región o del país que representan estos establecimientos (convenciones públicas).

Pero la tendencia reciente de mayor relieve es la importación de grandes volúmenes de bacalao fresco procedente de Rusia para cubrir el segmento de mercado de gama baja que opera bajo convenciones industriales y comerciales (establecimientos de menú diario, restauración colectiva) con un producto de menor precio y calidad para una cocina menos elaborada, pero destinada a un público mayoritario o a nuevos consumidores de pescado, caso de los jóvenes y los inmigrantes menos habituados a este producto y más atentos al precio como criterio de compra.

Por tanto, el sector del bacalao salado en Madrid aparece bifurcado entre el segmento de calidad, minoritario en tamaño, y la reciente entrada de nuevos 
productos más manejables para consumidores urbanos con poca motivación y tiempo para preparar la materia prima de forma tradicional y con escasos conocimientos que justifiquen la compra de los tipos de bacalao que presentan un precio más elevado.

\subsection{Cataluña}

Cataluña es uno de los principales mercados por su volumen demográfico y económico y por la demanda de productos de alta calidad. Estos factores han estimulado la producción regional, que ha ampliado su capacidad de transformado y distribución y también su área de mercado (cuadro 5). Esta expansión industrial y comercial ha tenido consecuencias muy significativas en la transformación de la coalescencia de convenciones en el resto de España, debido a la paulatina difusión de las convenciones imperantes en Cataluña (básicamente de tipo industrial y comercial) en otras áreas consumidoras y a la consiguiente sustitución o retroceso de algunas convenciones dominantes en otras regiones (Espinosa y Martínez, 2011a).

Este proceso de sustitución se explica por la complejidad creciente del mercado catalán del pescado salado durante la última década. Islandia se mantiene como primer proveedor de bacalao salado en Cataluña, seguida a distancia por proveedores secundarios como Rusia, China, Noruega y las Islas Feroe. La totalidad de los empresarios catalanes entrevistados trabajan con bacalao islandés, cuyas características comerciales de color blanco, gran anchura, lomos gruesos y humedad relativamente alta debido a la inyección de salmuera con fosfatos que retienen el agua en los tejidos del pescado (Gallart, Heide y Ostli, 2005) han sido muy bien aceptadas en Cataluña. Pese a la coexistencia de productores/distribuidores muy centrados en el mercado catalán con grandes empresas con ventas de escala nacional e internacional (Portugal, Italia), las entrevistas ponen de relieve el predominio de estas nuevas convenciones que han desplazado al modelo doméstico del bacalao nacional dorado de sabor intenso, menor grosor y prolongado proceso de curación.

En consonancia con este nuevo marco de convenciones, casi todos los productores catalanes han optado por elaborar su producto con inyección de salmuera y distribuirlo en grandes superficies de alimentación con representación a escala nacional. Solamente las empresas de menor tamaño se han enfocado en la modernización del mercado catalán del bacalao salado a través de la innovación y la oferta de proximidad. De hecho, la presencia de pequeños y medianos empresarios dedicados a la distribución y procesado del bacalao ad- 
quirido, por ejemplo, en el País Vasco, ha permitido el mantenimiento del Gremi de Bacalladers (gremio de bacaladeros), comerciantes y distribuidores de bacalao locales y regionales que han hecho de su oficio una artesanía que pervive hasta la actualidad y que representan una respuesta doméstica y local al modelo de negocio industrial.

En el seno de este canal de distribución, se ha logrado aumentar el valor añadido de un producto muy tradicional a través de la venta del bacalao desalado refrigerado, sin piel y sin espinas para su consumo inmediato, de la venta de bacalao remojado y ya desalado en agua en el punto de venta, o de la división por porciones individuales ya preparadas e incluso ya cocinadas. Con la introducción de productos preparados se ha incrementado el valor de las ventas al consumidor final y al sector de la restauración, muy favorecido por la simplificación del manejo de la materia prima.

\subsection{Comunidad Valenciana}

En esta región, el bacalao salado comparte protagonismo con otras salazones de pescado (sardinas, pulpo, caballa, atún) que se consumen en crudo (Gallart, Escriche y Fito, 2005). También se distingue esta Comunidad por la elaboración del bacalao llamado «de tipo inglés», que se obtiene lavando en agua el bacalao ya salado tras su proceso de curación y dejándolo de nuevo en curación por un tiempo determinado. El resultado es un bacalao menos salado, de color amarillo y sabor intenso, que se sirve crudo en pequeñas raciones como aperitivo, entrante o ingrediente para ensaladas.

La pervivencia de esta preparación tradicional ha generado unas convenciones domésticas muy arraigadas y casi exclusivas de esta región, donde uno de los productores entrevistados afirmaba: «nosotros no somos bacaladeros, sino salazoneros». Las empresas valencianas más artesanales prefieren el bacalao nacional por su idoneidad organoléptica para la preparación del tipo inglés. De hecho, es la única región donde no se ha ampliado el abanico de países proveedores y la más fiel al proceso productivo tradicional de salazón y secado, con limitada presencia de la salazón en salmuera o inyectada (cuadro 5).

Sin embargo, existe un problema creciente con el suministro de la materia prima adecuada para elaborar bacalao inglés. La creciente competencia en precios del bacalao de importación en el conjunto de España está generando un encarecimiento relativo del bacalao nacional que se hace particularmente notorio en esta región, carente de flota bacaladera. La reacción de algunos productores valencianos consiste en asumir estas nuevas convenciones comer- 
ciales y recurrir a la materia prima de importación, con color mucho más blanco, de mayor grosor y con una curación que no supera, en la mayoría de los casos, los cuarenta días.

En un intento por mantener intactas las propiedades del bacalao tipo inglés, un empresario entrevistado declaró: «estamos añadiendo pimentón y azafrán al bacalao blanco que compramos para que se parezca más al color dorado de antes». El consiguiente encarecimiento del producto final está limitando el consumo de salazones en Levante a ocasiones especiales y al nicho de consumidores de mayor poder adquisitivo, cuando se trataba de un artículo de consumo básico característico de la cultura alimentaria regional hace aún pocos años. Esta caída del consumo tradicional está empujando a las empresas valencianas a introducir las nuevas elaboraciones desaladas y fileteadas que también triunfan en las demás regiones estudiadas.

\section{DisCUSIÓN DE LOS RESUltAdOS REGIONALES: FACTORES DE CAMBIO Y PERMANENCIA} EN LA DEFINICIÓN DE CALIDAD

La teoría de las convenciones permite interpretar de manera coherente el conjunto de transformaciones que ha experimentado en la última década la concepción de la calidad del bacalao en salazón en los diferentes mercados regionales considerados.

La situación de partida puede calificarse como relativamente diversa. Sobre una tradición heredada de consumo de bacalao seco, salado y amarillento, bien suministrado por productores noruegos (Kurlansky, 1999 y Lauritzen, 2004), de los que España es el segundo cliente europeo tras Portugal (Xie y Myrland, 2010), bien elaborado en España con bacalao capturado por la flota vasca y gallega (Gago, 2007), se dibuja un mapa con ciertos contrastes internos: fidelidad al producto clásico en áreas de fuerte componente rural como Galicia y Andalucía, mayor originalidad en la Comunidad Valenciana por el apego a las salazones y la difusión local del tipo inglés, preferencia por las piezas grandes y algo más blancas en regiones de mayor poder adquisitivo como el País Vasco y Cataluña, más el eclecticismo multirregional de Madrid. En conjunto, imperaban las convenciones domésticas, definiéndose la calidad del bacalao salado en función de su origen geográfico y su elaboración y presentación tradicional.

Los factores mencionados en el apartado 3 han reducido esta variedad regional de gustos sobre el bacalao, como se ha puesto de relieve en el apartado 4. El resultado es un panorama más homogéneo. Los consumidores son ahora 
más sensibles a las convenciones industriales y comerciales incorporadas en las nuevas gamas de productos ofrecidos por la industria bacaladera y disponibles en los canales de venta minorista, tanto cadenas de supermercados como de hipermercados, que concentraron en 2012 el 60\% del volumen y del valor del pescado y el marisco vendidos en España, frente al 16\% de las tradicionales pescaderías especializadas, según la Base de Datos de Consumo en Hogares. La combinación de un mayor grado de procesamiento industrial y unos productos más manejables, asequibles y variados es patente en los filetes, los congelados, los desalados y los platos precocinados, que ganan cuota de mercado y en cuya elaboración no tiene tanto significado la calidad de la materia prima original.

Este proceso facilita la competencia de países como China, Rusia o Canadá, cuyos bajos costes y/o altos volúmenes de producción pueden abastecer con garantías las demandas de las factorías que a su vez surten a la gran distribución, extremadamente rigurosa en lo tocante a la homogeneidad y regularidad del contenido de sus secciones de refrigerados. Además, la citada innovación islandesa consistente en añadir fosfatos a la solución de salmuera inyectada hace que el bacalao retenga más agua y no adquiera color amarillento (Xie y Myrland, 2010; Sánchez, 2011b y Trondsen, 2012). Se obtiene así un producto semejante a los pescados frescos y blancos de más prestigio como la merluza (que concentra el $25 \%$ del volumen y del valor del pescado consumido en España en 2012, sin contar el marisco), a la vez que se incrementa su versatilidad industrial y su atractivo comercial, puesto que la frescura sigue siendo la cualidad más valorada por los consumidores españoles de pescado, pese al mayor precio medio que se paga por esta clase de alimento (cuadro 2).

El mutuo refuerzo entre las convenciones industriales y comerciales, propiciada por la entrada en escena de operadores que operan a gran escala en un marco institucional cada vez más liberalizado, se traduce en un retroceso de los demás criterios de definición de la calidad del bacalao, en particular de las convenciones domésticas. El descrédito de estas últimas no ha sido compensado desde Noruega con un esfuerzo de sus autoridades comerciales y compañías exportadoras por transformar el origen geográfico en un activo intangible, apelando a las convenciones públicas relacionadas con la notoria reputación del país como potencia pesquera (caso del salmón) o incluso a las ecológicas (por comparación de sus prácticas de pesca y elaboración naturales y sostenibles con el uso de fosfatos en el bacalao islandés y en un número creciente de empresas españolas). Incluso las emergentes convenciones cívicas, centradas en la contribución de los alimentos al bienestar social, juegan en contra de la tradición salazonera, puesto que las políticas sanitarias derivadas de las evi- 
dencias científicas disponibles acerca de las consecuencias negativas del consumo excesivo de sal propician el trasvase del consumo hacia los filetes al punto de sal.

No obstante, persisten elementos de contraste interregional que deben mencionarse y que, al menos de momento, limitan en algunas regiones la generalización de las convenciones industriales y comerciales como criterios casi exclusivos de evaluación de la calidad del bacalao en salazón.

En las regiones donde el comercio especializado y de proximidad conserva cierto significado se conservan las convenciones domésticas y cívicas. En Galicia, la dispersión del poblamiento y la pervivencia de modalidades tradicionales de abastecimiento alimentario como los mercados ambulantes permiten, junto con el arraigo popular del bacalao en salazón, que el gusto local no se haya transformado en la dirección dominante. En Cataluña, gracias a la actitud innovadora de los vendedores de bacalao al detalle, que actúan como auténticos centros de formación del consumidor en torno a la tradición bacaladera, la segmentación paulatina del mercado del bacalao en salazón puede entenderse como una reacción frente al aplastante predominio del bacalao blanco y grueso suministrado desde Islandia que ha copado la distribución organizada y el consumo más cotidiano. Dado que la protección al comercio minorista es una constante en Cataluña desde hace décadas (López, Lois y Fernández, 2013) bajo el argumento de su contribución a la vitalidad de las ciudades y sus barrios, la compra en estos establecimientos satisface dos relevantes criterios de definición de la calidad: producto tradicional (convenciones domésticas) y sostenimiento del entorno social y económico (conven- ciones cívicas).

En sentido contrario, donde estos factores territoriales y socioeconómicos están ausentes, caso de la Comunidad Valenciana, la elaboración tradicional encuentra dificultades para competir con los nuevos productos de base industrial-comercial y va quedando relegada a la marginalidad, porque la inadecuación de las materias primas importadas para elaborar el bacalao de tipo inglés hace que el producto pierda identidad y aceptación.

La restauración de calidad también funciona, en la práctica, como un reducto de las convenciones domésticas, a la vez que propicia el desarrollo de las convenciones públicas vinculadas a la reputación adquirida por determinados locales especializados en recetas tradicionales y en nuevas elaboraciones de bacalao en salazón. Las entrevistas con profesionales del sector sugieren que la procedencia geográfica del bacalao influye en sus decisiones de compra porque condiciona la calidad final de los platos y, a la vez, sirve como argumento o reclamo ante el público que frecuenta estos establecimien- 
tos. Se trata de un hecho constatado en el País Vasco, que ha hecho de la gastronomía un factor distintivo de su promoción turística y de su cultura popular y en la que el bacalao (al pil pil, a la vizcaína, al ajoarriero, en tortilla o en las más modernas brandadas) ocupa un lugar destacado. Los casos de Madrid y Cataluña reproducen este esquema, aunque con menor proyección pública.

\section{CONCLUSIONES}

En el plano conceptual, este artículo demuestra que la teoría de las convenciones puede aplicarse más allá de su uso habitual como marco explicativo de la creciente aceptación social de alimentos dotados de cierta singularidad, bien sea por su novedad y sostenibilidad (alimentos orgánicos), su procedencia geográfica (comercio justo, microproducciones locales) o su imagen pública (vinos de calidad). El bacalao en salazón, por el contrario, es un alimento de menor relieve, más genérico y por tanto susceptible de sustitución por otras elaboraciones del mismo pescado o por otras especies, en función de criterios como el precio, la manejabilidad, el aspecto o la salubridad. Sólo su arraigo histórico en la cultura gastronómica española le otorga posibilidades específicas de diferenciación que, precisamente, constituyen la base de su actual posición de mercado en las distintas regiones estudiadas. El recurso a la teoría de las convenciones en este contexto resulta novedoso respecto a la ya abundante literatura existente sobre temas agroalimentarios que se apoya en esta herramienta conceptual.

En el plano empírico, el principal resultado que se obtiene es la constatación de la bifurcación o dualización del mercado del bacalao en salazón. Las convenciones industriales y comerciales incorporadas en los filetes blanqueados al punto de sal elaborados en Islandia o en el género más barato procedente de Rusia, China y Canadá se han impuesto merced a las economías de escala, a la capacidad logística de la distribución minorista organizada y a los nuevos hábitos sociales de compra, preparación y consumo. En cambio, las convenciones domésticas y públicas respaldan la resistencia del bacalao noruego tradicional — seco, amarillento y muy salado- en los segmentos del mercado con mayor propensión a pagar precios superiores a cambio de productos de mayor calidad, consumidos o adquiridos en establecimientos que se distinguen por su reputación o proximidad. Las otras dos clases de convenciones, cívicas y ecológicas, apenas parecen manifestarse en este tipo de alimentos genéricos, al contrario de lo que la literatura consultada demuestra para los alimentos más diferenciados que se han citado. 
Ahora bien, este proceso de dualización tiene efectos regionales diferenciados que hacen posible la persistencia de una cierta diversidad en la definición del bacalao de calidad que impera en los distintos mercados estudiados. Es cierto que en todos los casos se observa la penetración del bacalao fileteado, desalado o precocinado, pero también se constata que en las regiones donde las condiciones territoriales (mayor componente rural), culturales (tradición culinaria arraigada y de reconocido prestigio), económicas (gran volumen de consumo y grupos sociales con cierto poder adquisitivo) lo permiten, el bacalao tradicional retiene una cuota de mercado mayor y, con ella, una imagen de producto más selecto avalada por su concentración en la restauración y el comercio especializado. En distinta proporción, según cada caso, estos factores se dejan sentir sobre todo en Galicia y el País Vasco, mientras en Cataluña y Madrid la bifurcación resulta muy evidente. La situación de la Comunidad Valenciana, donde el producto local tradicional se enfrenta a un futuro más incierto, debe entenderse precisamente como resultado de la ausencia o debilidad de estos factores complementarios.

En definitiva, los factores que, según Pitte (2001) modulan la «geografía del gusto», no pueden reducirse ya a la naturaleza y la cultura de cada continente, país o región, sino que debe reconocerse la influencia de los procesos económicos como vector de difusión de nuevas tendencias que, en todo caso, nunca sustituyen por completo a las preferencias alimentarias preexistentes, sino que se combinan con ellas para generar variantes territoriales específicas, más o menos diferenciadas entre sí en virtud de una serie de elementos que pueden ser analizados de forma integrada y coherente a la luz de la teoría de las convenciones.

Fecha de recepción: 29/10/2012

Fecha de aceptación: 30/05/2013

\section{Bibliografía}

Amilien, V., Forth, F. y Ferras, N. (2007): "Hyper-real territories and urban markets: changing conventions for local food - case studies from France and Norway". Anthropology of Food, Special Issue 2, 18 pp.

Boltanski, L. y Thévenot, L. (1991): De la justification. Les économies de la grandeur. París, Gallimard.

Consejería de Agricultura y Pesca (2009): Análisis de la producción pesquera regional. Sevilla, Junta de Andalucía. 
Díaz Méndez, C. y Gómez Benito, C. (2010): "Nutrition and the Mediterranean diet. A historical and sociological analysis of the concept of a "heathy diet" in Spanish society". Food Policy, 35, pp. 437-447.

Espinosa Seguí, A. y Martínez Alba, I. (2011a): Profiles of the Spanish salted fish markets. The Saltfish Project: Documento de Trabajo. Bergen, Departamento de Geografía, Universidad de Bergen.

Espinosa Seguí A. y Martínez Alba, I. (2011b): Regional market segmentation of a mature food product: The case study of salted codfish in Spain. The Saltfish Project: Documento de Trabajo. Bergen, Departamento de Geografía, Universidad de Bergen.

Gago González, J. M. (2007): El pequeño comercio en la posguerra castellana. De la cartilla de racionamiento a los supermercados. Valladolid, Junta de Castilla y León.

Galdós, R. (2004): "La intervención pública en la promoción de la calidad agroalimentaria: normativa comunitaria, española y vasca”. Investigaciones Geográficas, 34, pp. 47-62.

Gallart Jornet, L., Escriche Roberto, I. y Fito Maupoey, P. (2005): La salazón del pescado, una tradición en la dieta mediterránea. Valencia, Universidad Politécnica de Valencia.

Gallart Jornet, L., Heide, M. y Ostli, J. (2005): "Market situation of Norwegian bacalao on the Mediterranean Spanish coast". Okonomisk Fiskeriforskning, 15, pp. 51-55.

Gallart Jornet, L., Rodríguez-Barona, S., Barat, J. M. y Fito, P. (2003): "Elaboración y comercialización del bacalao salado". Alimentaria, 348, pp. 87-94.

García Orellán, R. (2006): "Rumbo al Gran Banco: una etnohistoria de la pesca industrial del bacalao en los bancos de Terranova". Revista Internacional de Estudios Vascos, 51, pp. 577-592.

García Santos, R. (2007): El bacalao en la cocina vasca. San Sebastián, Iano Books.

González López, M. (2011): "The institutions-innovation dilemma: the case of coastal fisheries". Journal of Technology Management E Innovation, 6/3, pp. 184-195.

Grafe, R. (2004): "Popish habits vs nutritional need: feasting and fish consumption in Iberia in the Early Modern Period". Discussion Papers in Economic and Social History, 55. Oxford, University of Oxford.

Guthey, G. T. (2008): "Agro-industrial conventions: some evidence from northern California's wine industry". The Geographical Journal, 174, pp. 138-148.

Kirwan, J. (2006): "The interpersonal world of direct marketing: examining conventions of quality at UK farmers' markets". Journal of Rural Studies, 22, pp. 301-312.

Kurlansky, M. (1999): El bacalao. Biografía del pez que cambió el mundo. Barcelona, Ediciones Península.

Lagendijk, A. (2004): "Global 'lifeworlds' versus local 'systemworlds': how flying winemakers produce global wines in interconnected locales". Tijdschrift voor Economische en Sociale Geografie, 95, pp. 511-526.

Lauritzen, K. (2004): Quality of salted cod (Gadus morhua L.) as influenced by raw material and salt composition. Dr. Scient Thesis. Norwegian College of Fishery Science. Tromso. 
Lindkvist, K. B., Gallart Jornet, L. y Stabell, M. C. (2008): "The restructuring of the Spanish salted fish market”. The Canadian Geographer, 52, pp. 105-120.

Lindkvist, K. B. y Lois González, R. (2008): "Aspectos regionales de las pesquerías de Galicia y Noruega”. Eria, 51, pp. 69-77.

Lindkvist, K. B. y Sánchez Hernández, J. L. (2008): “Conventions and innovation: a comparison of two localised natural resource-based industries". Regional Studies, 42, pp. 343-354.

López González, A., Lois González, R. y Fernández Casal, R. (2013): “Mercadona (Spain): a retail model in expansión". International Journal of Retail \& Distribution Management, 41, pp. 6-26.

Martínez Alba, I. y González Romero, G. (2012): "Estrategias empresariales en la industria de la salazón de bacalao en Andalucía". Espacio, Tiempo y Forma. Revista de Ciencias Humanas, 26, pp. 27-37.

Murdoch J., Marsden, T. y Banks, J. (2000): "Quality, nature, and embeddedness: some theoretical considerations in the context of the food sector". Economic Geography, 78, pp. 107-125.

Morris, C. y Kirwan, J. (2007): "Is meat the new militancy? Locating vegetarianism within the alternative food economy", en D. Maye, L. Holloway y M. Kneafsey (eds.): Alternative food geographies. Representation and practice. Amsterdam, Elsevier, pp. 133-145.

Murdoch, J. y Miele, M. (1999): “Back to nature': changing 'worlds of production' in the food sector". Sociologia Ruralis, 39, pp. 465-483.

Parrott, N., Wilson, N. y Murdoch, J. (2002): "Spatializing quality: regional protection and the alternative geography of food". European Urban and Regional Studies, 9, pp. 241-261.

Pitte, J. R. (2001): "La géographie du goût, entre mondialisation et enracinement local". Annales de Géographie, 621, pp. 487-508.

Ponte, S. (2009): "Governing through quality: conventions and supply relations in the value chain for South African wine". Sociologia Ruralis, 49, pp. 236-257.

Ponte, S. y Gibbon, P. (2005): "Quality standards, conventions and the governance of global value chains". Economy and Society, 34, pp. 1-31.

Pudup, M. B. (2008): "It takes a garden: cultivating citizen-subjects in organized garden projects". Geoforum, 39, pp. 1228-1240.

Renard, M. C. (2003): "Fair trade: quality, market and conventions". Journal of Rural Studies, 19, pp. 87-96.

Rosin, Ch. (2008): "The conventions of agri-environmental practice in New Zealand: farmers, retail driven audit schemes and a new spirit of farming". GeoJournal, 73, pp. 45-54.

Rosin, Ch. y Campbell, H. (2009): "Beyond bifurcation: examining the conventions of organic agriculture in New Zealand". Journal of Rural Studies, 25, pp. 35-47.

Sánchez Hernández, J. L. (2009): "Redes alimentarias alternativas: concepto, tipología y adecuación a la realidad española". Boletín de la Asociación de Geógrafos Españoles, 49, pp. 185-207. 
Sánchez Hernández, J. L. (2011a): "The food value chain as a locus for (dis)agreement: conventions and qualities in the Spanish wine and Norwegian salted-cod industries". Geografiska Annaler: Series B, Human Geography, 93, pp. 105-119.

Sánchez Hernández, J. L. (2011b): The Norwegian salted cod value chain facing the Spanish seafood market: explanations for a disagreement. The Saltfish Project: Documento de Trabajo. Departamento de Geografía, Universidad de Bergen.

Sánchez Hernández, J. L., Aparicio Amador, J. y Alonso Santos, J. L. (2010): "The shift between worlds of production as an innovative process in the wine industry in Castile and Leon (Spain)". Geoforum, 41, pp. 469-478.

Storper, M. (1995): "The resurgence of regional economies: the region as a nexus of untraded interdependencies". European Urban and Regional Studies, 2, pp. 191-221.

Storper, M. (1997): The regional world. Territorial development in a global economy. Nueva York, The Guilford Press.

Trondsen, T. (2012): "Value chains, business conventions, and market adaptation: A comparative analysis of Norwegian and Icelandic fish exports". The Canadian Geographer / Le Géographe canadien, 56/4, pp. 459-473.

Wilkinson, J. (2002): "The final foods industry and the changing face of the global agro-food system". Sociologia Ruralis, 42, pp. 329-346.

Xie, J. y Myrland, O. (2012): "Modeling market structure of the Spanish salted fish market". Food Economics-Acta Agriculturae Scandinavica, Section C/7, pp. 119-127.

\section{Sitios web}

FAO (Organización de las Naciones Unidas para la Alimentación y la Agricultura). Disponible en: http://faostat3.fao.org/home/index.html\#HOME (Fecha de consulta: 05/10/2012).

Ministerio de Agricultura, Alimentación y Medio Ambiente [en línea]. Base de datos de consumo en hogares. Disponible en: http://www.magrama.gob.es/es/alimentacion/temas/consumo-y-comercializacion-y-distribucion-alimentaria/panel-de-consumo-alimentario/base-de-datos-de-consumo-en-/ogares/consulta10.asp (Fecha de consulta: 29/07/2013).

Ministerio de Economía y Competitividad [en línea]. Datacomex - Estadísticas del comercio exterior. Disponible en: http://datacomex.comercio.es/ (Fecha de consulta: 05/10/2012).

Ministerio de Agricultura, Alimentación y Medio Ambiente [en línea]. Estudio del Consumo Alimentario Extradoméstico en España. Hábitos del Consumidor. Conclusiones. Disponible en: http://www.magrama.gob.es/es/alimentacion/temas/consumoy-comercializacion-y-distribucion-alimentaria/OTO\%C3\%91O_INVIERNO_2011 _tcm7-207355.pdf (Fecha de consulta: 05/10/2012). 


\section{RESUMEN}

Este artículo estudia las transformaciones recientes en los criterios que productores, distribuidores y consumidores utilizan para definir y juzgar la calidad del bacalao en salazón en las regiones españolas. Conforme al marco conceptual de la teoría de las convenciones, se distinguen hasta seis grupos de criterios diferentes: industriales, comerciales, domésticos, públicos, cívicos y ecológicos. Los datos estadísticos oficiales y, sobre todo, las entrevistas semiestructuradas con los actores integrantes de la cadena de valor en una muestra representativa de regiones españolas permiten afirmar que las convenciones domésticas, relacionadas con el origen geográfico y la preparación tradicional del bacalao en salazón, están dejando paso a las convenciones industriales y comerciales como criterio principal de definición de la calidad. No obstante, la diversidad regional en la apreciación del bacalao pervive en segmentos del mercado como la restauración de calidad y el comercio especializado, más sensibles a los valores de tradición, proximidad y reputación.

PAlabras Clave: bacalao salado; calidad; pautas de consumo; convenciones por regiones; innovación alimentaria; España.

\section{AbSTRACT}

This article analyzes recent changes in the criteria used by producers, wholesalers, retailers and consumers to define and assess the quality of salted cod in a sample of Spanish regions. In accordance with the conceptual framework of the theory of conventions, six types of criteria are taken into account: industrial, market, domestic, public, civic, and ecological. Statistical data supplied by food authorities and semistructured interviews with actors in the value chain provide empirical evidence enabling us to state that domestic conventions, related to geographical origin and traditional salted cod recipes, are increasingly challenged by industrial and market conventions as the main arguments for assessing quality. Nevertheless, regional diversity is still apparent in specific market niches like restaurants and specialized retailing, where values of tradition, proximity and reputation remain influential.

KEY WORDS: salted codfish; quality; consumption patterns; regional conventions; food innovation; Spain.

\section{RÉSUMÉ}

Cet article étudie les transformations récentes des critères que les producteurs, les distributeurs et les consommateurs utilisent pour définir et juger la qualité de la morue salée dans les régions espagnoles. Conformément au cadre conceptuel de la théorie des conventions, on peut distinguer jusqu'à six groupes de critères différents : industriels, commerciaux, domestiques, publics, civiques et écologiques. Les données statistiques officielles et, surtout, les entretiens semi-structurés avec les acteurs qui intègrent la chaîne de valeur dans un échantillon représentatif des régions espagnoles permettent d'affirmer que les conventions domestiques, en rapport avec l'origine géo- 
graphique et la préparation traditionnelle de la morue salée, sont en train de céder leur place aux conventions industrielles et commerciales comme critère principal de définition de la qualité. Cependant, la diversité régionale dans l'appréciation de la morue existe toujours dans certains segments du marché comme la restauration et le commerce spécialisé, plus sensibles aux valeurs de tradition, proximité et réputation.

Mots Clé: morue; qualité; modes de consommation; conventions régionales; innovation alimentaire; Espagne. 